\title{
Realidad aumentada como tecnología aplicada a la educación superior: Una experiencia en desarrollo
}

\author{
Mario Badilla Quesada', Ana María Sandoval Poveda²
}

1. Producción electrónica multimedial (PEM), Universidad Estatal a Distancia (UNED); mbadilla@uned.ac.cr 2.Producción de materiales didácticos y escritos (PROMADE), Universidad Estatal a Distancia (UNED); amsandoval@uned.ac.cr

\author{
Recibido: 11 de junio del 2015 Corregido: 03 de setiembre del 2015 Aceptado: 16 de setiembre del 2015
}

\begin{abstract}
Resumen
El estudio forma parte del trabajo realizado por la Red de investigación para el desarrollo de conocimientos y propuestas tecnológicas innovadoras aplicables a la educación superior a distancia de la Universidad Estatal a Distancia (UNED) de Costa Rica, sobre el tema de la Realidad Aumentada (RA). El trabajo comprende tanto el estado del arte como las acciones concretas dentro del ámbito universitario en este campo. El objetivo del material es mostrar las bases del trabajo con RA desarrollado en los últimos años y presentar el proceso de evolución de esta tecnología como potenciadora de la comunicación, así como su utilidad para la innovación educacional.
\end{abstract}

Palabras clave: tecnología, educación, comunicación, inovación educacional, aprendizaje, realidad aumentada

\section{REALIDAD AUMENTADA (RA)}

Hay ciertas tecnologías que surgen de forma experimental, restringidas a entornos de especialistas investigadores, pero que con el tiempo se vuelven más accesibles, tanto para productores de contenidos y desarrolladores como para los consumidores. Una de estas tecnologías es la Realidad Aumentada (RA), se habla y escribe mucho acerca de ella $y$, debido a que marca una tendencia, probablemente se continúe utilizando en nuevos estudios y propuestas.

La RA es una variación de los ambientes virtuales o realidad virtual, como se le llama comúnmente. La

\section{Abstract \\ Augmented reality as applied technology to higher education: an experience in development}

This research is part of the work done by the Research Network to Develop Knowledge and Innovative Technological Solutions Applicable to Higher Distance Education of the Universidad Estatal a Distancia (UNED) of Costa Rica in the field of Augmented Reality (AR). This work includes the state of the art as well as specific actions within the university's scope. The goal of this material is to show the foundations of the work related to AR done in the last few years, and to introduce the evolving process of this technology as communication enhancer and its utility for educational innovation.

Key words: technology, education, communication, edcational innovations, learning, augmented reality

realidad virtual es una experiencia completamente digital e inmersiva que reemplaza el mundo real por uno simulado; según Pérez (2011, p. 4), la realidad virtual es "la percepción en 3D de entornos simulados que permiten trasladar al usuario a mundos de ensueño y le posibilitan viajar a través del tiempo al pasado y al futuro". Mientras esto sucede, el usuario está inmerso en un ambiente artificial y no puede ver a su alrededor.

En contraste, la RA se entiende como "la generación de imágenes nuevas a partir de la combinación de información digital en tiempo real y el campo de visión de una persona" (Johnson et al. 2013, p. 12). La RA se puede considerar como una mezcla entre lo completamente artificial y lo completamente real. 
La RA también puede ser utilizada para "borrar" información de la realidad, en vez de agregarla, como se muestra en la aplicación Transporter Room que recrea el transportador de la serie de ciencia ficción Star Trek. Esta aplicación remueve un objeto físico del visor del dispositivo móvil. Algunos le dan el nombre de Realidad Disminuida (E-Axis.com, 2012). A partir de la definición básica y la descripción de las capacidades de la RA se enumeran tres características necesarias para contar con verdadera RA; según Kipper y Rampolla (2012):

- Debe combinar información real y virtual.

- Requiere ser interactiva en tiempo real.

- Debe operar y ser usada en un ambiente en tercera dimensión.

La RA, muy similar a otras interfaces gráficas, brinda la habilidad de trasferir información útil al espectro visual, en tiempo real y en cualquier lugar; es la combinación de varias tecnologías que trabajan en conjunto para trasladar la información digital a la percepción visual. Gene Becker, citado por Kipper y Rampolla (2012), indica que la RA es muchas cosas, entre ellas: una tecnología, un campo de investigación, una visión del futuro en la computación, una industria comercial emergente y un nuevo soporte para la expresión creativa.

De acuerdo con las definiciones, también es posible señalar lo que no es RA. Existen en la actualidad muchos ejemplos de medios mejorados que no necesariamente constituyen ejemplos de una realidad "aumentada". Un fotomontaje hecho con Adobe Photoshop @ $\mathrm{u}$ otro software de edición no es RA, aunque pueda activarse con alguna aplicación usada para ello. Tampoco incluye cine ni televisión, aunque tengan gráficos realistas generados por computadora mezclados con video, no son interactivos, por lo tanto no son RA. Los códigos QR tampoco se consideran RA, aunque de cierto modo se activan de manera similar, reconociendo una imagen, pero no combinan simultáneamente la información virtual con la realidad.

Código QR (abreviatura de quick response code) es la marca registrada para un tipo de código de barras de matriz (o bidimensional). El código consiste de módulos negros (puntos rectangulares) organizados en un patrón, de forma cuadrada, sobre un fondo blanco. La información codificada puede estar conformada por datos, principalmente de tipo numérico y alfanumérico (Neal, 2012). Para procesar un código QR, se necesita un dispositivo con capacidad de rastreo de imagen (la mayoría de smartphones o teléfonos inteligentes poseen esta funcionalidad). Una vez rastreado el código, la persona usuaria es redirigida a un sitio web, un documento, un video o cualquier material que haya sido creado para asistirlo, en el caso de los usos educativos, en su proceso de aprendizaje (ver imagen 1)

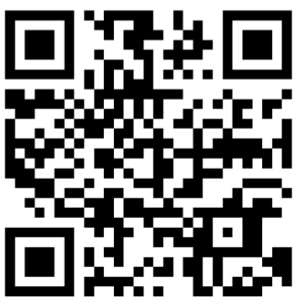

Imagen 1. Código QR. Fuente: Generado en qrpedia.org

\section{ANTECEDENTES}

"Aumentar" la realidad no es una idea nueva ni en el área conceptual, ni en la tecnológica. Existen indicios importantes, aunque rudimentarios, que mostraban el potencial de esta tecnología. Se pueden enumerar algunos ejemplos que se enmarcan dentro de este concepto:

- Sensorama (1962). Fue creado por el cinematógrafo Morton Heilig. Consistía en una máquina que buscaba abarcar todos los sentidos por medio de una actividad que el espectador recibía frente a una pantalla, en la cual se proyecta una película con imágenes estereoscópicas 3D y la máquina producía viento y aromas (Payatagool, 2008).

- The Ultimate Display (1965). Propuesto por el científico Ivan Sutherland. Constaba de un "casco" con lentes semitransparentes que proyectaban datos. Se podría considerar el precursor de los actuales lentes de realidad aumentada y el Google Glass (Sutherland, 1965). Ese último dispositivo fue retirado del mercado por la compañía Google, hasta que puedan desarrollar una versión más pulida y asequible de las gafas "inteligentes". Dougherty (2015) menciona que actualmente el proyecto Glass es más un caso de estudio por los problemas del hardware en desarrollo cuyo propósito no está claro. 
- Video Place (1988). Proyecto propuesto por el artista digital Myron Krueger. Utilizaba la tecnología para rastrear las siluetas de personas y generar efectos, el resultado se proyectaba en una pantalla como si esta fuera un lienzo (Camejo, s.f.).

Estos ejemplos, producto de amplias investigaciones y del ingenio de sus creadores, muestran precedentes y casos de estudio, y por qué no, de inspiración para la RA, ya que según diferentes especialidades se presentan soluciones técnicas diversas, pero con similitudes conceptuales principalmente en el hecho de enriquecer y mediar la forma de ver la realidad.

A Tom Caudell y a David Mizell, investigadores de la compañía aeronáutica Boeing, se les atribuye el haber acuñado el término Realidad Aumentada en 1992 Esto es fruto de su trabajo de investigación y desarrollo, el cual se centraba en encontrar una manera de facilitar el proceso ingenieril y de manufactura, la cual los llevó a diseñar un software que pudiera sobreponer, sin hacerlo realmente, las posiciones de ciertos cables; esto durante el proceso de construcción (Kipper y Rampolla, 2012).

\section{¿Cómo funciona?}

Existen conceptos y elementos básicos para entender cómo funciona la RA en la actualidad, los cuales delinean los componentes necesarios en distintas plataformas. Respecto al hardware se requiere una computadora o un dispositivo móvil, una pantalla, una cámara y un marcador (geolocalización, reconocimiento de imágenes). En el caso del software es preciso contar con una aplicación informática o programa para RA, y un servidor de contenidos.

La RA se puede presentar de dos formas: reconociendo una imagen marcador o mediante un punto de localización geográfica, por eso al señalar que se requiere un marcador se brindan las dos opciones.

Cuando se utiliza un marcador básicamente se asocia un modelo virtual en tercera dimensión a un objeto físico; cuando se usa la localización, en lugar de reconocer un marcador, se asigna información digital a un grupo de coordenadas geográficas.

El proceso de RA por medio de un marcador o a partir de una imagen comienza por una cámara que muestra una señal de video en tiempo real, la señal es digitalizada e interpretada por el programa que, a su vez, identifica el marcador y lo asocia con el contenido digital asignado a él y, finalmente, el contenido digital es reproducido dentro del marco de la señal de video a través de la pantalla del dispositivo o el monitor de la computadora (ver imagen 2).

Deben existir claves o señales en el ambiente para que la cámara pueda posicionar el contenido digital correctamente. Estas señales pueden ser características naturales en el ambiente o colocadas artificialmente, como se aprecia en el ejemplo de la imagen 2. Para

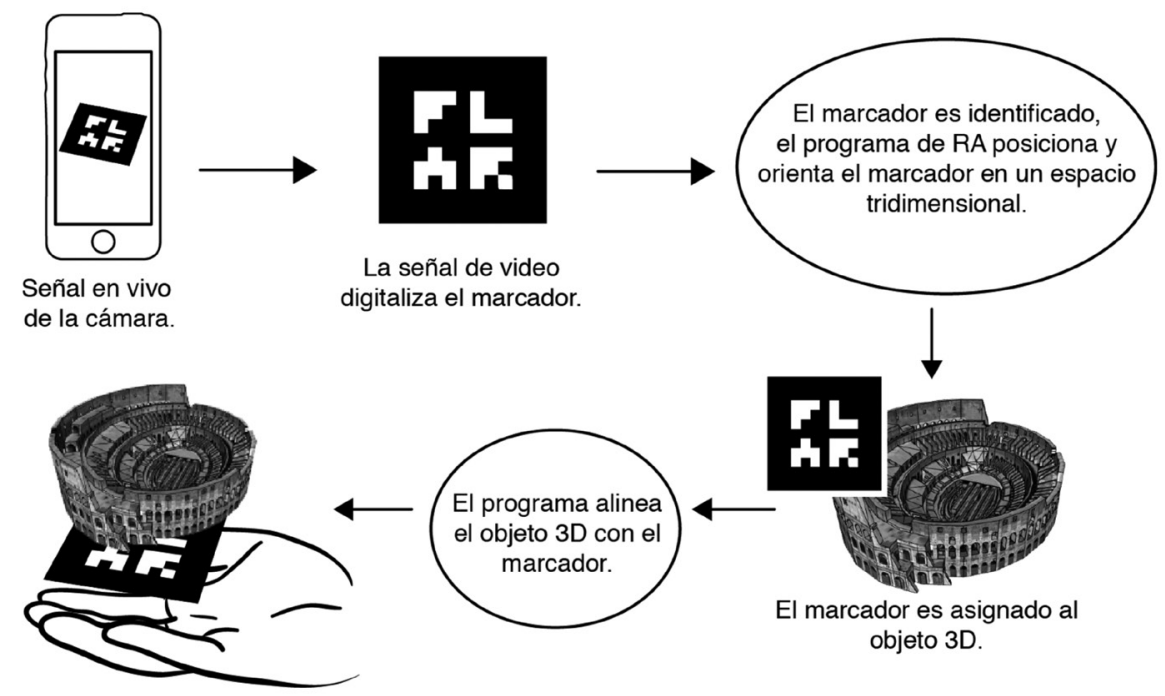

Imagen 2. Proceso del funcionamiento de RA. Fuente: Elaborado a partir de Kipper y Rampolla (2012) 
hacer la RA más simple, muchas aplicaciones utilizan una imagen como marcador; las imágenes utilizadas específicamente para este objetivo se llaman también marcadores fiduciales o símbolos fiduciales (Craig, 2013). Este tipo de marcador hace más fácil el proceso automático de rastreo y de identificación de puntos de referencia para el alineamiento del contenido digital.

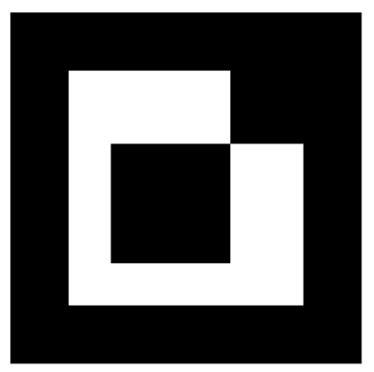

Imagen 3. Ejemplo de marcador fiducial o bidimencional. Fuente: Generado en qrpedia.org

Uno de los retos de la RA es el reconocimiento de los marcadores citados debido a lo variable que es el mundo físico en comparación a los laboratorios o los ambientes de pruebas. Con el avance de la tecnología, estos marcadores han evolucionado, así de ser simples formas geométricas en blanco y negro a imágenes con más detalle y color.

En el caso de la RA geoposicionada, el marcador está dado por el sistema de posicionamiento global (GPS, por su nombre en inglés global positioning system) del dispositivo móvil que se use. GPS es un sistema de navegación que utiliza una red de satélites en el espacio. El receptor puede calcular la ubicación en los ejes X y Y, y potencialmente $Z$ (Craig, 2013). Esta ubicación puede ser almacenada en una base de datos. Posteriormente, una aplicación de RA puede hacer uso de esta información y mostrarla espacialmente en relación con la cámara del dispositivo. Esta ubicación geográfica funciona como un marcador o seña natural en el ambiente.

En general, este tipo de marcador permite ampliar la información de una zona, reconstruir parajes antiguos, recrear momentos históricos e incluso añadir información del lugar destinada a diferentes públicos (estudiantes, turistas o participantes en una actividad programada, entre otros).

\section{PRESENTE}

La RA ha tenido un gran repunte en los últimos años debido al uso de teléfonos móviles inteligentes (smartphones) y tabletas (tablets), debido a que muchas aplicaciones hacen uso de la cámara y la pantalla integradas en el dispositivo para mostrar datos mezclados con el mundo real.

Una de las primeras aplicaciones desarrolladas para emplear la tecnología citada es Layar, la cual es también una de las más utilizadas. Este programa o aplicación genera capas de contenido que se eliminan o agregan. Los contenidos pueden ser alimentados por los usuarios a partir de las redes sociales, como por ejemplo con el uso de Twitter, Foursquare, Google Maps, entre otros. Otras aplicaciones similares a Layar son Acrossair, Wikitude y Tagwhat, entre otras.

En algunos casos, el uso de RA tiene fines meramente de entretenimiento, comunicación o lucro, pero una de sus funciones reales es asistir al usuario en el proceso de toma de decisiones.

La RA, además de su aplicación en la industria del entretenimiento, posee un alto potencial para ser utilizada en entornos educativos, principalmente para favorecer el aprendizaje y la evaluación del conocimiento adquirido. Tal como lo indica Marc Prensky, el estudiantado de hoy no solo necesita que lo que aprenda sea relevante, sino que sea real, que tenga una aplicación y pueda ser empleado de manera inmediata en situaciones reales (Prensky, 2011). Lo anterior es posible al permitir que la acción sobre los elementos RA le habilite para lograr un contacto diferente con su objeto de estudio. Con la ayuda de esta tecnología podrán esclarecerse conceptos complejos.

En el caso específico de la educación a distancia, su uso se reviste de otras utilidades, ya que se trabaja para fomentar el autoaprendizaje (UNED, 2004). Esta tecnología permite una interacción más cercana a la realidad entre el estudiante y el contenido, así como también puede mostrar ambientes enriquecidos de una situación en el lugar de estudio, o exponer un objeto lejano en el espacio real en que se encuentra. Además, implica una participación activa de los estudiantes, quienes pasan más tiempo con el material educativo, lo cual mejora la experiencia y potencia la efectividad del aprendizaje. 
El Informe Horizon de 2011, publicado en 2012, señala la relevancia de la adopción de esta tecnología para la docencia, el aprendizaje o la investigación creativa:

- Posee un gran potencial para proveer tanto aprendizaje contextual in situ como la exploración espontánea y el descubrimiento de las conexiones naturales de la información con el mundo real.

- Con su implementación, los estudiantes que visiten sitios históricos pueden acceder a aplicaciones de RA que sobreponen información y mapas que muestran cómo se veía el lugar en diferentes momentos de la historia.

- La creación de juegos basados en el mundo real puede aumentarse con datos conectados a la red. Esto proporcionará al cuerpo docente nuevas y poderosas formas de mostrar correlaciones y conexiones y a la comunidad estudiantil nuevas maneras de acercarse al conocimiento.

El Informe Horizon de 2010, centrado en Iberoamérica, indica que esta tecnología se puede aprovechar en casi cualquier disciplina o área de estudio, pero principalmente en las relacionadas con las ciencias aplicadas (Ingeniería, Química, Física y Biología), así como en la Cirugía, la Arqueología y la Museología, entre otras.

Por ejemplo, el American Museum of Natural History, de Nueva York, tiene sus propios elementos de RA disponibles para explorar y descubrir el sitio y para múltiples exposiciones en las diferentes salas (American Museum of Natural History, s.f.). Además, en su página de internet cuentan con un espacio dedicado a las aplicaciones del museo, entre las cuales se incluye la RA.

\section{RA en la UNED}

Particularmente, en la Universidad Estatal a Distancia (UNED) de Costa Rica ya se han dado pasos para aplicar la RA en diferentes espacios y momentos.

El trabajo con RA en la institución comenzó desde 2011 y un grupo de investigadores de la Red de investigación para el desarrollo de conocimientos y propuestas tecnológicas innovadoras aplicables a la educación superior a distancia trabajan en su utilización en diferentes cursos que se ofertan en la universidad.
El empleo de elementos de esta naturaleza se encuentra respaldado por el modelo pedagógico de la institución, el cual establece claramente que el estudiante es el centro del proceso educativo de forma que tenga "la libertad de aprovechar al máximo los recursos que se le ofrecen, de planificar el progreso de su aprendizaje y de regular por sí mismo, el ritmo y la calidad de sus avances" (UNED, 2004, p. 13). Para lograr esto, es necesario implementar cambios en las formas de aprendizaje y el diseño de las propuestas pedagógicas usadas en la educación a distancia. Además, al estar la RA como una de las doce tecnologías en vigilancia tecnológica, según el Informe Horizon (2013), se justifica que sea una de las que se integren primero a estas tareas (ver imagen 4).

La experiencia vivida en la UNED comenzó en la Vicerrectoría de Investigación; específicamente en el Observatorio de Tecnología en Educación a Distancia (proyecto que pertenece a la Red de investigación mencionada anteriormente). Allí, un grupo de investigadores trabajó los dos primeros ejemplos, ambos muy básicos y consistentes únicamente en movimientos que el usuario puede ejecutar para ver elementos en 3D desde diferentes ángulos. Los ejemplos están disponibles en el sitio del proyecto. El primero corresponde al concepto de esfera y sus elementos, relacionado con conceptos matemáticos (Universidad Estatal a Distancia, s.f.b); el otro al Coliseo romano, acorde con el libro de Historia del arte que se utiliza en el curso de Estudios Generales (Universidad Estatal a Distancia, s.f.a). En ambos casos, debe imprimirse una hoja con el marcador e ingresar a un enlace para activar el programa correspondiente. En estos casos, se trabaja con una computadora con acceso a internet y cámara web disponible.

A partir del interés generado, se han construido materiales educativos para promover el conocimiento de esta herramienta y sus posibles usos educativos. El Observatorio, ha elaborado vodcast, publicado novedades y estudiado diferentes aplicaciones para la RA, entre ellos:

- El vodcast Realidad Aumentada y sus aplicaciones en educación está disponible al público en general y ha sido visto numerosas veces (Hernández, 2013).

- La nota Mesa de realidad aumentada con Kinect (Badilla, 2012b) muestra un ejemplo de aplicación de la RA.

- Unos meses después, llegó la información titulada El futuro del teclado y el mouse (Badilla, 2012a). 


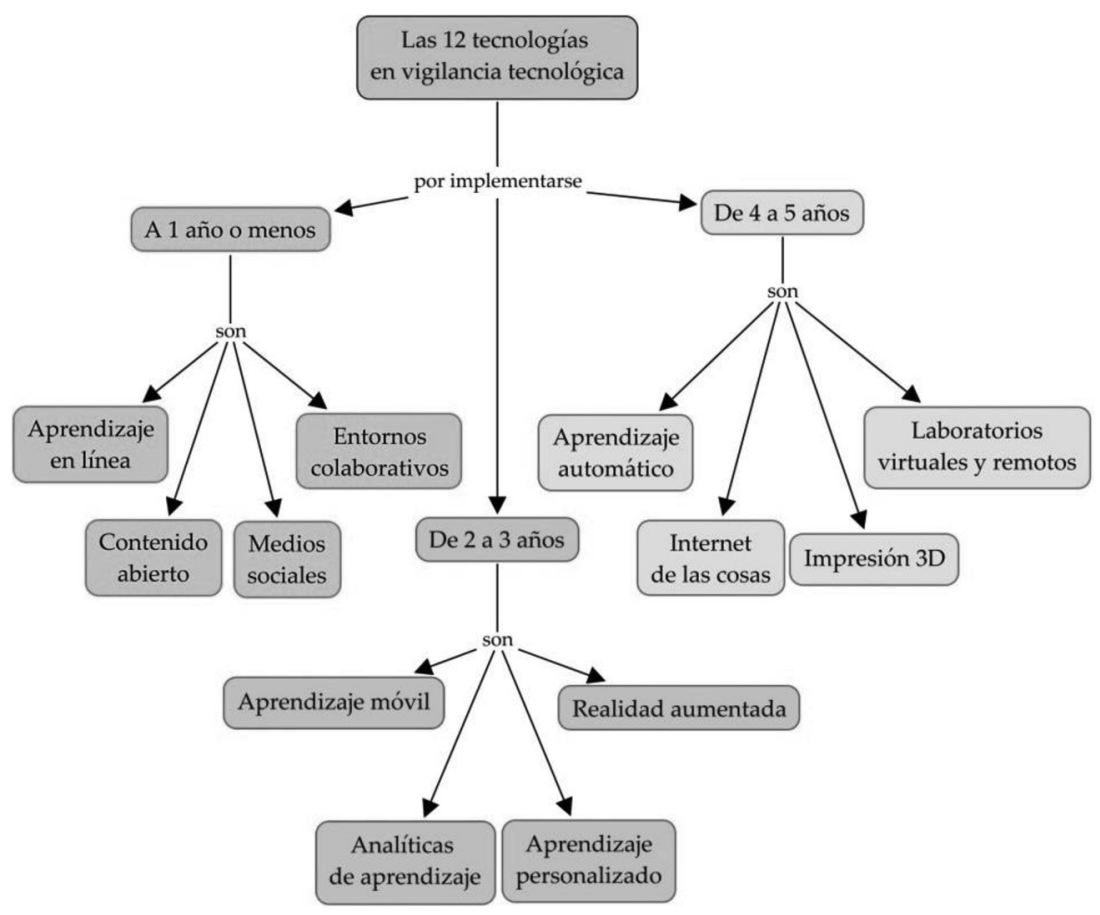

Imagen 4. Tecnologías en vigilancia tecnológica. Fuente: Elaborado a partir de Johnson et al., 2013.

- Respecto a las aplicaciones de RA para tecnología móvil, se hizo un aporte con las siguientes publicaciones: ¿Cómo puede Layar contribuir a la educación? (Badilla, 2014) y Una App para realidad aumentada: Aurasma (López, 2014). Específicamente, porque ya se trabajaba en un proyecto piloto de utilización de este tipo de aplicaciones junto con los materiales didácticos de la UNED.

- Los dos últimos aportes sobre este tema se publicaron respecto a desarrollos o tecnologías trabajadas por Google, estas son Posibilidades de Google Glass en educación (Badilla, 2014c) y Google invierte en Magic Leap (Badilla, 2014b). La primera incluye una infografía con 30 opciones para la aplicación al tema sugerido y la segunda una pequeña muestra de la combinación de la tecnología con la vista humana.

Por otra parte, mostrar los trabajos realizados hasta el momento ha provocado reacciones internas y externas a la universidad. Es evidente que el tema ha sido objeto de atención académica y también mediática; especialmente por la utilización de la tecnología en ámbitos educativos y científicos.

- Internamente, estudiantes de la UNED, participantes del proyecto Protored, entrevistaron a la coordinadora del Observatorio, Diana Hernández, y publicaron en su blog información sobre el trabajo que se realiza en la UNED en RA (Proto-Red San Carlos, 2012).

- $\quad$ El 28 de agosto de 2014, el periódico de distribución nacional, La Nación, publicó una nota acerca del proyecto que utiliza la RA en los materiales didácticos de Anatomía y Fisiología Humanas I (Vargas, 2014). En este artículo se incluye uno de los ejemplos piloto que se mostró en el encuentro de Ciencia Ciudadana de ese año.

- En esas mismas fechas FollowAR publicó la misma nota en su sitio de Facebook. FollowAR es una aplicación para RA que mantiene informados a los usuarios interesados en lo novedoso sobre el tema de RA.

- Remarqus e Iberoamerica.net también compartieron la publicación de La Nación en sus sitios web.

Finalmente, el proceso de trabajo con RA llevó a la puesta en marcha de un plan piloto con la Escuela de Ciencias Exactas y Naturales, específicamente con la del programa Registros y Estadísticas de Salud. En este caso se trabajó con dos elementos de RA y un video, los cuales se ponen a disposición de los estudiantes 
en la unidad didáctica del curso Anatomía y Fisiología Humanas I, a partir del tercer cuatrimestre de 2015.

\section{VISTAZO AL FUTURO}

Las posibilidades que abre el uso creativo de la tecnología en la educación son incontables; muchas realizables de manera inmediata y otras a futuro.

En el caso de la RA el panorama no es menos alentador, la constante actualización de los dispositivos propicia que exista una mayor relación usuario-interfaz y permite que cada vez más personas tengan acceso a las tecnologías, las cuales aumentan y enriquecen la vida profesional, académica y cotidiana.

En los próximos cinco años, debería haber cambios significativos en la tecnología de RA, y se puede esperar que se integre más a la vida cotidiana. Ya hay indicios de esto.

Algunas editoriales han publicado libros con elementos de RA, tal es el caso de la Editorial Saldaña con sus materiales sobre el Cuerpo humano, Civilizaciones y Dinosaurios; cada uno con seis elementos de RA consistentes principalmente en juegos interactivos.

También la Editorial Costa Rica hizo su parte al agregar este tipo de elementos a la novela Un fuego lento de Jessica Clark.

En ambos casos, las editoriales elaboraron su propia aplicación para ver e interactuar con los elementos y la pusieron a disposición de los lectores en forma gratuita.

En el ámbito educativo, también hay propuestas que se llevan a cabo. En la UNED ya se cuenta con varias solicitudes de uso de esta tecnología para cursos de diferente naturaleza.

Adicionalmente, el Movimiento Maker ya ha comenzado a florecer dentro del estudiantado y eso permitirá, a futuro, utilizar su labor para enriquecer los elementos de RA que se elaboren en la universidad, a la vez que la RA podrá contribuir con los objetivos que estos grupos se planteen (Lee, 2012).

El Movimiento Maker o Do It Yourself (DIY) consiste en una serie de iniciativas destinadas a que las personas aprendan cómo funcionan las cosas desde el punto de vista del constructor, de manera que se posible apropiarse de los procesos, las técnicas y la tecnología existentes detrás de cualquier tarea humana (Sarasa, 2014); en la UNED esto se encuentra en gestación y ya los mismos estudiantes se encargan de capacitar a otros para que se incluyan en esta labor.

En un futuro cercano, se usará para aprender a reparar equipos, para recibir información turística directo en el lugar de interés o para observar cómo se cambian las tintas de la impresora sobre el mismo aparato.

En un futuro remoto, los dispositivos no serán más que lentes de contacto y podremos visualizar información personal de los transeúntes mientras se camina por la calle, esto gracias al reconocimiento de rostros y la computación en la nube. Ahí está el verdadero reto: el mundo físico se mezclará con el digital y, en lugar generar asombro, el efecto natural será el beneficiarnos por su uso.

\section{CONCLUSIONES}

Los elementos de RA son cada vez más comunes en la sociedad: publicidad, ingenierías, medicina y otras áreas del saber aprovechan sus potencialidades para hacer llegar el mensaje deseado. Lo anterior permite comprender por qué es una tecnología en vigilancia tecnológica (Johnson et al., 2013) y también por qué es una de las que la UNED ha elegido para trabajar. Por esta razón, se considera que la labor debe continuar, pues el estudiantado de las diferentes carreras se beneficiará de su aplicación en las distintas áreas del saber.

Como herramienta tecnológica, la RA presenta sus propias características, a la vez, sus aplicaciones son amplias, tal como lo indican Kipper y Rampolla (2012). En la UNED el aspecto relacionado con la investigación ya se considera y se trabaja activamente; queda pendiente el trabajo de expresión creativa y de visión del futuro, pero se espera que al abrir el uso de RA se planteen proyectos que permitan abarcar estos ámbitos.

La RA impactará a quienes busquen confeccionar sus recursos y de igual manera sucederá si solamente se utilizan, lo cual se plantea desde hace algunos años (Lee, 2012); es tarea de la UNED facilitar esas interrelaciones en ambos sentidos, de manera que se enriquezcan ambos grupos de personas.

La situación actual es muy interesante, en general la tecnología avanza rápidamente en el sector de la educación y los estudiantes adoptan las innovaciones 
de forma intuitiva con nuevas habilidades cognitivas y flexibilidad para aprender.

En la UNED, por medio de los proyectos que se mencionaron anteriormente, se ha indagado en el alcance y el funcionamiento de esta tecnología. A medida que más áreas del conocimiento se incorporen a este tipo de esfuerzos será posible comprender qué tipo de contenidos son más susceptibles a presentarse por medio de RA.

Otra de las formas de continuar trabajando con la tecnología en mención, es incorporar metodologías de investigación que permitan determinar su eficacia en los procesos de aprendizaje a distancia. Los prototipos que se mencionan en esta investigación y futuros materiales pueden servir para realizar pruebas con el público meta de la universidad, por ejemplo, ensayos guiados, encuestas, observación de experiencias, entre otros.

Este tipo de pruebas servirán para obtener información cualitativa que retrate el uso de RA como herramienta de aprendizaje y las necesidades específicas tanto de la población estudiantil como de la academia. Por lo demás, se puede potenciar el aprendizaje independiente a distancia y crear una participación más activa de los estudiantes.

En el contexto de la UNED, una motivación implícita de implementar una tecnología es que los académicos y los responsables de tomar decisiones conozcan las herramientas con que cuentan para enriquecer la labor formativa. Asimismo, es necesario democratizar el conocimiento para educar en el uso de tecnologías, no como un fin, sino como un medio óptimo para reforzar los procesos de enseñanza y aprendizaje.

\section{REFERENCIAS}

American Museum of Natural History. (s.f.). Bernard Family Hall of North American Mammals. Recuperado de: http://www.amnh.org/apps/ bernard-family-hall-of-north-american-mammals

Badilla, M. (2012a). El futuro del teclado y el mouse. Observatorio de tecnología en educación a distancia. Recuperado de: http://observatoriotecedu.uned.ac.cr/ el-futuro-del-teclado-y-el-mouse/.

Badilla, M. (2012b). Mesa de Realidad Aumentada con Kinect. Observatorio de tecnología en educación a distancia.
Recuperado de: http://observatoriotecedu.uned.ac.cr/ mesa-de-realidad-aumentada-con-kinect/.

Badilla, M. (2014a). ¿Cómo puede Layar contribuir a la educación? Observatorio de tecnología en educación a distancia. Recuperado de: http://observatoriotecedu.uned. ac.cr/webinar-uso-de-layar-en-educacion/.

Badilla, M. (2014b). Google invierte en Magic Leap. Observatorio de tecnología en educación a distancia. Recuperado de: http://observatoriotecedu.uned.ac.cr/ motion-leap-recibe-inversion-google/.

Badilla, M. (2014c). Posibilidades de Google Glass en educación. Observatorio de tecnología en educación a distancia. Recuperado de: http://observatoriotecedu.uned. ac.cr/posibilidades-de-google-glass-en-educacion/.

Camejo, M.P. (s.f.). Myron Krueger [entrada de blog]. Recuperado de: https://elartedigital.wordpress.com/ artistas/myron-krueger/.

Craig, A.B. (2013). Understanding augmented reality: concepts and applications. Waltham: Elsevier Science.

Dougherty, C. (18 de enero, 2015). A retreat for Google Glass and a case study in the perils of making hardware [entrada de blog]. Recuperado de: http://bits.blogs.nytimes. com/2015/01/18/a-retreat-for-google-glass-and-a-casestudy-in-the-perils-of-making-hardware/.

E-Axis.com. (27 de marzo de 2012). Star Trek Augmented Reality (iOS) [Video]. Recuperado de: http://www.youtube.com/watch? $\mathrm{v}=9 \mathrm{ES} 6 \mathrm{aS} 82 \mathrm{~K} 2 \mathrm{k}$.

García, I., Peña-López, I., Johnson, L., Smith, R., Levine, A. y Haywood, K. (2010). Informe Horizon: edición Iberoamericana 2010. Austin, Texas: The New Media Consortium.

Hernández, D. (Productora). (12 de agosto de 2013). Realidad Aumentada y sus aplicaciones en educación [Video podcast]. Recuperado de: https://www.youtube.com/ watch?v=jplQ0Cuz9q4.

Johnson, L., Adams, S. y Cummins, M. (2012). Informe Horizon del NMC: Edición para la enseñanza universitaria 2012. Austin, Texas: The New Media Consortium.

Johnson, L., Adams Becker, S., Gago, D., García, E. y Martín, S. (2013). NMC perspectivas tecnológicas: educación superior en América Latina 2013-2018. Un análisis regional del informe Horizon del NMC. Austin, Texas: The New Media Consortium.

Johnson, L., Smith, R., Levine, A. y Stone, S. (2010). The 2010 Horizon Report: Edición en español. (Xavier Canals, Eva Durall, Translation.) Austin, Texas: The New Media Consortium. Recuperado de: http://www.nmc.org/ pdf/2010-Horizon-Report-es.pdf. 
Kipper, G. y Rampolla, J. (2012). Augmented reality: an emerging technologies guide to AR. Massachusetts: Syngress.

Lee, K. (2012). The future of learning and training in augmented reality. InSight: A Journal of Scholarly Teaching, 7, 31-42. Recuperado de: http://files.eric.ed.gov/fulltext/ EJ980168.pdf.

López, G. (2014). Una App para realidad aumentada: Aurasma. Observatorio de tecnología en educación a distancia. Recuperado de: http://observatoriotecedu.uned.ac.cr/ una-app-para-realidad-aumentada-aurasma/.

Neal, B. (2012). Scan and Learn: Using QR Codes as Part of Your Learning Solution. T+D, 66(10), 92-93.

Payatagool, C. (septiembre, 2008). Theory and research in $\mathrm{HCl}$ : Morton Heilig, pioneer in virtual reality research. Recuperado de: http://www.telepresenceoptions. com/2008/09/theory_and_research_in_hci_mor/.

Pérez, F.J. (marzo, 2011). Presente y futuro de la tecnología de la realidad virtual. Creatividad y sociedad. n. ${ }^{\circ} \mathrm{XVI}$. Recuperado de: https://www.academia.edu/5081919/ Francisco_Javier_P\%C3\%A9rez_Mart\%C3\%ADnez_ Presente_y_Futuro_de_la.

Prensky, M. (2011). Enseñar a nativos digitales. Madrid: Ediciones SM, Biblioteca innovación educativa.

Proto-Red San Carlos. (2012). Realidad aumentada [entrada de blog]. Recuperado de: https://protoredsancarlos.wordpress.com/2012/02/06/realidad-aumentada/.
Sarasa, D. (2014). Creatividad tecnológica: mantener al alcance de los niños. Economía aragonesa. Recuperado de: http://www.gorilaa.com/resources/o6loOSw1mk/4c36a 85ab285190fe9cf27244f7daaec.pdf\#page=95.

Sutherland, I. (1965). The ultimate display. Recuperado de: http://worrydream.com/refs/Sutherland\%20-\%20 The\%20Ultimate\%20Display.pdf.

Universidad Estatal a Distancia. (2004). Modelo pedagógico. San José: Vicerrectoría académica. Recuperado de: http://www.uned.ac.cr/conuniversitario/images/docs_ cu/politicas_academicas/ModeloPedagogico_000.pdf.

Universidad Estatal a Distancia. (s.f.a). Realidad aumentada. Coliseo romano. San José: Observatorio de tecnología en educación a distancia. Recuperado de: http://investiga.uned.ac.cr/ra/coliseo.html.

Universidad Estatal a Distancia. (s.f.b). Realidad aumentada. Concepto: esfera. San José: Observatorio de tecnología en educación a distancia. Recuperado de: http://investiga.uned.ac.cr/ra/esfera.html.

Vargas, M. (28 de agosto de 2014). Alumnos de la UNED aprenden con "realidad aumentada". La Nación. Recuperado de: http://www.nacion.com/vivir/ciencia/AlumnosUNED-aprenden-realidad-aumentada_0_1435656434. html. 
